\title{
Higher order aberrations of the eye: Part two
}

\section{Authors:}

Marsha Oberholzer

Wayne D.H. Gillan²

Alan Rubin ${ }^{2}$

\section{Affiliations:}

${ }^{1}$ Department of Optometry, University of the Free State,

South Africa

${ }^{2}$ Department of Optometry, University of Johannesburg, South Africa

\section{Corresponding author:} Marsha Oberholzer, oberholzerm@ufs.ac.za

\section{Dates:}

Received: 05 Nov. 2015 Accepted: 24 Feb. 2016 Published: 24 June 2016

How to cite this article: Oberholzer M, Gillan WDH, Rubin A. Higher order aberrations of the eye: Part two. Afr Vision Eye Health. 2016;75(1), a335. http:// dx.doi.org/10.4102/aveh. v75i1.335

Copyright:

(C) 2016. The Author(s). Licensee: AOSIS. This work is licensed under the Creative Commons Attribution License.
This article is the second in a series of two articles, which provides a discussion of the factors that may possibly contribute to variable results when wavefront aberrations of the human eye are measured. Some of the factors discussed in this article are the influences that refractive errors (specifically myopia and astigmatism), pupil diameter, accommodation of the crystalline lens, age, mydiatric drops and the integrity of the tear film may have on these wavefront measurements. The first article in the series explained the general principles of higher order aberrations (HOAs), as well as HOAs of importance in the eye and the measuring apparatus used to measure HOAs of the eye.

\section{Introduction}

This article is the second in a series of two articles reviewing the factors as discussed by researchers that contribute to variable results when measuring higher order aberrations (HOAs) of an eye. The first article largely explained the general and fundamental principles that apply to HOAs, as well as indicated exactly which HOAs are of importance in the eye and also the measuring apparatus commonly used to measure HOAs of the eye.

\section{Factors influencing the measurement of higher order aberrations of the human eye}

Some variables influencing the measurement of HOAs have been noted by researchers in the past. These variables need to be carefully evaluated when measurements of wavefront aberrations (WAs) are taken on subjects and especially when the measurements of different subjects are compared. Some of these factors are refractive error, contrast sensitivity function (CSF), pupil diameter, accommodation of the crystalline lens, age, mydiatric drops and the integrity of the tear film. Although this list of variables is not complete, the more important factors that need to be considered are discussed in this article.

\section{Myopia and astigmatism}

Hyperopia could possibly be included in this section of the article; however, the research on which this article is based was mostly on myopia and astigmatism. The literature available on WAs and hyperopia is somewhat limited, and thus, this review will discuss only myopia and astigmatism.

Paquin et al. ${ }^{1}$ reason that myopia is a visual wavefront aberration (WA) or imperfection that affects a great section of the population. Almost $30 \%$ of people (20-40 years of age) are myopic, rising from a prevalence of about $2 \%$ in 6-year-old children to about $20 \%$ in 20 -year-old adults. Saw et al. ${ }^{2}$ define myopia as a state of refraction when light focuses in front of the retina of an unaccommodated eye and is clinically associated with blurred distance vision, rubbing of the eye and peering. The physiologic classification of myopia entails the gradual increase of the axial length of the eye and the pathological classification, the abnormal lengthening of the eye. Atchison et al. ${ }^{3}$ proposed three models of myopic elongation, namely equatorial elongation or peripheral expansion, posterior pole or central elongation and, lastly, global expansion which aids in the understanding of the characteristics of lower order aberrations (LOAs) and HOAs present in myopes. Detailed explanations of the elongation models may be found elsewhere. ${ }^{3}$

Factors causing myopia are mostly the curvature of the cornea, the crystalline lens surfaces, the refractive index gradient of the crystalline lens and axial elongation of the eye with increases in the anterior and vitreous chamber depths. Both genetics and environmental factors are widely accepted as playing a role in the development of myopia. Myopia is thus a multifactorial problem

Note: This article is based on doctoral studies for of the first author with the supervision of Professors W.D.H. Gillan and A. Rubin of the University of Johannesburg. The degree, DPhil, was conferred on 25 March 2015. 
with many contributing factors including axial elongation, surface component power and environmental influences. ${ }^{4}$

Atchison ${ }^{5}$ and Atchison et al. ${ }^{3}$ suggest that the most important optical defects for normal eyes are refractive errors, and after compensation of the refractive errors, residual HOAs are possibly the only issue remaining. Reduced visual performance in fully corrected myopes may thus be due to the presence of HOAs within these eyes. ${ }^{3,6}$ Previous literature $^{7,8}$ has shown that HOAs may influence myopia development, especially spherical aberration (SA). Atchison ${ }^{7}$ suggests that SA changes at a rate of $\pm 0.007 \mu \mathrm{m}$ per dioptre (D) of myopia and that the anterior cornea contributes the most to HOAs in relation to the total HOAs of the eye. WAs further change more slowly as myopia increases. Positive SA is usually present when the eye is in a relaxed accommodative state, and as the eye accommodates, the positive SA reduces. ${ }^{8}$ After two to three dioptres of accommodation, this positive SA shifts into negative SA due to the change in shape and refractive index distribution of the crystalline lens. SA is thus a key parameter for establishing the sign for defocus based on image contrast. ${ }^{8}$ The authors further argue that positive $\mathrm{SA}$, in combination with myopic blur and an overpowered eye, produces a monotonic decline in image contrast as the spatial frequency increases, whereas the opposite is true in the case of hyperopic blur and an underpowered eye. A myopic eye that is not corrected with positive SA and that rarely engages in near work has a weak inhibition to eye growth and will continue to grow resulting in an even more myopic eye. Hyperopic blur is thus a risk factor for myopia progression if the eye has negative SA. Similarly, positive SA would protect the eye against myopia progression and possibly prevent myopia. ${ }^{8}$

A study investigating astigmatism, ${ }^{9}$ reports that an association between infantile against-the-rule astigmatism and schoolage myopia exists whereas Parnissen ${ }^{10}$ reports the possibility of an association between astigmatism (against-the-rule) and the progression of myopia in children. Tian et al. ${ }^{4}$ researched the role of HOAs and retinal image degradation in the development of myopia and found that corneal astigmatism and cylindrical refractive errors are well correlated. Eyes with more astigmatism seem to have deeper anterior chambers and eyes with more myopia seem to have more positive SA, but the total HOAs of these eyes do not show any increase with higher myopia. The authors concluded that spherical refractive error, SAs and cylindrical refractive error may all contribute to myopic development. Kwan et al. ${ }^{11}$ studied WAs in myopic eyes compared to that of non-myopic eyes as well as that of more myopic eyes to less myopic eyes. For the first comparison (myopic vs. non-myopic eyes), the results showed significant differences between the axial lengths of the groups. No significant differences could be found with regard to total HOAs (3rd and 4th order HOAs), 3rd order HOAs, 4th HOAs or SA. However, with linear regression analysis, the slopes of 4 th order HOAs and SA were significantly different from zero. The second comparison (more myopic vs. less myopic eyes) showed that the axial lengths of the more myopic eyes were significantly longer compared to the axial lengths of the less myopic eyes. The more myopic eyes had statistically significantly lower total HOAs, 3rd order HOAs and SA compared to the less myopic eyes. SAs may thus be negatively correlated with myopia and more myopic eyes tend to have smaller amounts of SA. Contradictory results were however found by Karimian et al. ${ }^{12}$ who suggested that there is no significant correlation between SA and the degree of myopia.

The studies of Wang and Koch, ${ }^{13}$ Porter et al. ${ }^{14}$, CastenjónMochón et al. ${ }^{15}$ and Carkeet et al. ${ }^{16}$ observed that the HOAs between the right and left eyes of patients were correlated, and thus the reason for Wei et al. ${ }^{17}$ only using right eyes with pupil diameters of $6 \mathrm{~mm}$ in their particular study. Wei et al. ${ }^{17}$ investigated the HOAs of right eyes in 166 Chinese subjects with a mean age of $32.1 \pm 6.2$ years. Aberrometry measurements on these subjects were analysed, and correlations between HOAs, myopia, astigmatism and age were investigated. The results indicated that the 3rd order root-mean-square (RMS) contributed $49 \%$ towards the total RMS (3rd - 5th order), with the 4th order RMS contributing $39 \%$ and the 5 th order RMS, only $12 \%$. A slight statistical significant correlation was found between myopia and trefoil $\left(C_{3}^{3}\right)$, as well as between astigmatism and coma $\left(C_{3}^{1}\right)$ and trefoil $\left(C_{3}^{3}\right)$. He et al. ${ }^{18}$ performed a 4 -way analysis of variance (ANOVA) between the groups of children or adults with either myopia or emmetropia and found that the differences in RMS Zernike coefficients between the emmetropic and myopic adults were significant for all six orders of aberrations (2nd - 7th orders). The RMS Zernike coefficients in myopes were further found to increase significantly with an increase in refractive error, and the authors stated that an RMS Zernike coefficient of $2.5 \mu \mathrm{m}$ induces the same image degradation as $0.75 \mathrm{D}$ of blur. Two contradicting studies showing no correlation between myopia and HOAs are those of Levy et al. $^{19}$ and $\mathrm{Li}$ et al. $^{20}$ Levy et al. ${ }^{19}$ found no significant difference between the mean values of HOAs, total SA, total coma and total trefoil aberrations for right and left eyes. The results of the study by $\mathrm{Li}$ et al..$^{20}$ showed that nearest equivalent sphere (NES) has no significant correlation with the RMS values of HOAs (3rd - 5th order), SA and coma. Atchison et al. $^{3}$ found that myopia may be accompanied by only moderate or no increase in HOAs and this concurs with the results of studies done by other scientists such as Buehren et al..$^{6}$ and He et al. ${ }^{18}$ which present evidence that WAs do not increase significantly with an increase in myopia. Contrary studies $^{3,21}$ have, however, found moderate increases in WAs with an increase in myopia. Atchison et al. ${ }^{3}$ investigated the higher order RMS values and did not find any significant relationship with myopia although they reported that similar studies have found moderate increases in WAs with myopia. They also found SA to have a mean positive value that was significantly different from zero. Carkeet et al. ${ }^{21}$ suggest that myopia may be accompanied with high levels of HOAs and may result in poorer retinal image quality, even with the appropriate spectacle correction. They investigated 273 Singapore school children (male and female) with high myopia, low myopia, emmetropia and hyperopia. The HOAs 
with the largest mean average significantly different from zero (with $t$-testing) were found to be vertical and horizontal coma $\left(C_{3}^{-1}\right.$ and $\left.C_{3}^{1}\right)$ and SA $\left(C_{4}^{0}\right)$. The low myopes showed less positive $\mathrm{SA}$ compared to the other refractive groups used in this study. A similar finding was made by Collins et al. ${ }^{22}$

Studies addressing asymmetrical blur related to cylinder are not easily found. Many studies investigate myopia and HOAs, but few studies address the cylinder component of the refractive error with HOAs. Cheng et al. ${ }^{23}$ measured the aberrations of 200 eyes of 100 subjects with dilated pupils (mean pupil diameter of $7.58 \mathrm{~mm}$ ) and paralysed accommodation in six groups. A tendency for the highly astigmatic eyes $(>|-1|$ D) to have higher levels of HOAs compared to that of the low-astigmatic and non-astigmatic eyes was found. Astigmatic eyes also tend to have slightly larger total HOAs RMS values that were statistically significant with an independent $t$-test. No significant correlation was, however, found between refractive error and HOAs.

Hartwig and Atchison ${ }^{24}$ published a paper where they analysed the HOAs of a large clinical population of 24000 subjects (taken from the total sample of 40850 used for the original study). The study investigated the correlations present between the aberrations measured and three variables namely distance refraction, near addition and cylinder. The results of the investigation showed that the aberration coefficients were significantly dependent on the named variables, where SA accounted for $12 \%$ of the aberrations, horizontal coma for $9 \%$ and the HOA RMS Zernike coefficients for $7 \%$. Near addition was found to contribute the most to horizontal coma $(8.5 \%-9.0 \%)$, and NES was the greatest contributor to SA $(7.7 \%-11.6 \%)$. The results of their study ${ }^{24}$ further showed that SA becomes more positive as the spherical equivalents of the subjects become less negative or more positive, and thus, it is expected for higher myopes to have more negative SA. Aberration coefficients were also found to be significantly affected by refraction, especially with regard to horizontal coma and SA that accounted for $2.8 \%$ and $7.7 \%$ of the variation, respectively. The researchers thus found that most 3rd and 4th order HOAs were significantly dependent on NES, near addition and cylinder.

To summarise the very complicated influences of myopia on HOAs, the developmental studies of myopia have shown that HOAs such as SA may have an impact on myopia and may even be a causative factor for myopia., ${ }^{8,25}$ Some authors have found no increase in the total HOAs with an increase in myopia. ${ }^{3,46,12,18,20,23}$ Kwan et al. ${ }^{11}$ showed that no statistical difference exists in WAs between three groups (high myopes, moderate myopes and non-myopes) with regard to total HOAs (3rd and 4th order), 3rd order HOAs, 4th order HOAs or SA, but they did find that fourth order HOAs and SA was significantly different from zero and may thus have a great impact on the total HOAs of the eye. Other authors found a positive relationship between the variables of myopia and HOAs. Examples here include Collins et al. ${ }^{22}$ and Carkeet et al. ${ }^{21}$ who found that myopes presented with statistically lower 4 th order HOAs, specifically positive SA, compared to that of emmetropes. He et al. ${ }^{18}$ found higher RMS Zernike coefficients and 4th order HOAs for myopic adults compared to emmetropic adults, while Wei et al. ${ }^{17}$ found a statistically significant correlation between myopia and trefoil $\left(C_{3}^{3}\right)$ as well as between astigmatism and coma and trefoil $\left(C_{3}^{1}\right.$ and $\left.C_{3}^{3}\right)$. Hartwig and Atchison ${ }^{24}$ reason that many myopes have negative SA rather than positive SA and a decrease in pupil diameter may also reduce positive SA. Cheng et al. ${ }^{23}$ further showed higher degrees of HOAs in eyes with more astigmatism $(>|-1| D)$ compared with eyes with lower or no astigmatism.

\section{Contrast sensitivity function}

A paper by Campbell and Green ${ }^{26}$ deals mainly with the CSF affected by monochromatic aberrations and therefore, more information regarding the CSF affected specifically by chromatic aberrations may be found elsewhere. ${ }^{26}$ Williams et al. $^{27}$ argue that CSF is improved when most WAs are reduced with a deformable mirror or adaptive optics (AO) system, compared to when only defocus and astigmatism (LOAs) are corrected. Feizi and Karimian ${ }^{28}$ investigated the relationship between HOAs and CSF in 70 myopic eyes. The 'Area Under The Log Contrast Sensitivity Function' (AULCSF) was calculated for all 70 eyes, and the results of the study showed that the AULCSF was negatively correlated with the cycloplegic NES, the total RMS Zernike coefficients, as well as the RMS Zernike coefficients for SA. Feizi and Karimian ${ }^{28}$ concluded that SA significantly affects the CSF in myopic eyes. Wang et al..$^{29}$ investigated the relationship between HOAs and low contrast visual acuity (VA) in myopic eyes under mesopic conditions and found that SA is significantly associated with low contrast VA.

\section{Pupil diameter}

The pupil of the eye plays a major role in how much light enters the eye and, together with refractive state, also influences the image quality at the retina. ${ }^{30}$ When light is interrupted by an aperture, the result is the scattering and diffraction of light rays with less diffraction for a greater pupil diameter; however, more WAs will be induced in the optical system. The human eye is not diffraction-limited for pupil sizes greater than $1 \mathrm{~mm}$ and the overall best pupil diameter for transverse imaging over spatial frequencies from 0 to 30 cycles/degree (c/deg) is usually between $2 \mathrm{~mm}$ and $3 \mathrm{~mm}$ provided vision is corrected. ${ }^{27,30,31,32}$ The Zernike polynomial description of WAs depends on the pupil area analysed. Therefore, pupil diameter and consequently pupil area are of great importance when measuring WAs. ${ }^{32}$ Links between the pupil and retina, such as distance, position, shape and size of the pupil, are critical factors, and these links may be used to reduce the relative importance of diffraction and WAs on the image quality. ${ }^{31}$ 
Rodríguez et al. ${ }^{33}$ concluded from the results of their study that pupil diameter and pupil alignment are the most critical factors in the accuracy of aberrometry measurements. Burakgazi et al. ${ }^{34}$ found that WAs change significantly with a change in the size and position of the measuring pupil and Ginis et al. ${ }^{35}$ found that the WF measurement variation was higher near the margins of the pupil. A study by Dobos et al. ${ }^{36}$ found that the magnitude of the Zernike coefficients and their corresponding 95\% limits of agreement (LoA) increased with an increase in pupil diameter and that the pupil diameter and alignment of the aberrometer when taking measurements are thus of utmost importance when comparing different eyes and the WAs thereof. In agreement with Smolek ${ }^{37}$ and Ginis et al. ${ }^{35}$, Dobos et al. ${ }^{36}$ also suggest that when comparing different eyes the pupil diameters need to be the same (or within a small range of diameters) to ensure accuracy of comparisons of the measurements of WAs. Dobos et al. ${ }^{36}$ also found that under cycloplegic conditions the mean NES shifts more myopically with an increase in pupil diameter. With an increase in pupil diameter $(3 \mathrm{~mm}-7 \mathrm{~mm})$, the impact on HOAs was found to increase from $2.7 \%$ to $13.8 \%$ by CastejónMochón et al. ${ }^{15}$; thus there is a direct relation between total RMS Zernike coefficients and the diameter of the pupil.

\section{Accommodation}

López-Gil et al. ${ }^{38}$ investigated the relationship between age and accommodation-related changes in WAs of patients aged 19-60 years. The authors of the study concluded that WAs change with accommodation and with age, and He et al. ${ }^{18}$ and Williams et al. ${ }^{27}$ also agree that greater WAs are present in an accommodated eye. The increased WAs in patients older than 50 years may be explained by optical changes in the crystalline lens and reduced SA as a result of pupil miosis. Similar studies were conducted by Atchison et al. ${ }^{39}$ and Collins et al. ${ }^{22}$ with regard to accommodation. The findings of the two studies correlate with each other and those above as both indicated that SA becomes more negative as accommodation increases. In a letter to the editor of a peerreviewed journal it was noted by the writers, Awwad et al. ${ }^{40}$, that a decreasing pupil diameter causes SA to become negligible, and the authors found that negative SA increases with accommodation which may be explained by the accommodation-induced change of the crystalline lens shape, position and refractive index gradient as a whole. Thibos et al. ${ }^{8}$ also investigated accommodation and HOAs and found that positive SA is usually present in an unaccommodated eye, and with accommodation positive SA decreases and negative SA increases after two to three dioptres of accommodation. They attributed the change from positive to negative accommodation, similarly to Awwad et $\mathrm{al}^{40}$, to changes in the shape and refractive index distribution of the lens.

\section{Age}

Atchison and Markwell ${ }^{41}$ investigated 106 subjects aged between 18 and 69 years. The effect of age on the RMS Zernike coefficients of the ocular aberrations, the RMS Zernike coefficients of corneal aberrations and total RMS Zernike coefficients of the aberrations were investigated. The ocular RMS Zernike coefficients of the aberrations showed a significant increase in the HOAs with age in the 4th and 5th orders. The only coefficient that changed significantly with age was that of horizontal coma $\left(C_{3}^{1}\right)$ and this change may be due to changes in lens like tilt and decentration. The means of a few coefficients, however, were found significantly different from zero as well. The corneal RMS Zernike coefficients of the aberrations also showed an increase of HOAs with age; however, this increase was only significant for the 6th order, and no coefficient varied significantly with age. The last aspect investigated, the total RMS Zernike coefficients of the aberrations, increased significantly with age. Jahnke et al. ${ }^{42}$ conducted a similar study and found that an increase in age caused an increase in WAs and thus also in RMS Zernike coefficients of the 3rd and 4th order HOAs. Coma showed a tenfold increase and SA a twofold increase with increasing age. This increase in WAs may be exaggerated by other factors such as changes in crystalline lens. Wei et al. ${ }^{17}$ investigated the HOAs in a myopic Chinese population and also identified a statistically significant correlation between SA and age. However, the study by Levy et al. ${ }^{19}$ showed no correlations between SA, coma or trefoil aberrations and age. Possible differences between the studies that may account for the difference in results is that Jahnke et al. $^{42}$ had a sample size of 98 eyes which included myopes, emmetropes and hyperopes with age ranging between 17 and 65 years, but Levy et al. ${ }^{19}$ used a sample size of 70 eyes of subjects with socalled supernormal vision (VA $\geq 20 / 15)$ ranging between 18 and 51 years.

Hartwig and Atchison ${ }^{24}$ conducted an analysis on a large clinical population with a total sample size of 40850 subjects of which 13038 cases had near additions ranging from $0.75 \mathrm{D}$ to $4.0 \mathrm{D}$. They found that all 3rd order HOAs, SA and secondary SA (6th order WA) were significantly affected by the near addition, especially for horizontal coma and SA aberrations. They also argued that other changes of the ocular lens, such as the surface curvature, asphericities, centre thickness and refractive index distribution, are caused due to increase in age.

\section{Mydriatic drops}

Taneri et al. $^{43}$ investigated the influence of mydriatic eye drops on aberrometry measurements taken with the Zywave $^{\mathrm{TM}}$ aberrometer in terms of the predicted phoropter refraction (PPR) and HOAs. A mydriatic dilated group of eyes were compared with a group of non-dilated eyes. The results of the study showed that the PPR values were less myopic in the mydriatic dilated group compared with the undilated group. Changes in the RMS Zernike coefficients and SA between the groups were significantly different. The authors suggest that measurements be done without mydriasis to minimise refractive surprises in relation to possible refractive surgery. Tabernero et al. ${ }^{44}$ also argue that pharmacologic pupil dilation may account for centration variability as a result of shifts in the pupil centres with respect 
to natural conditions which may in turn have an effect on the generation of coma aberrations.

\section{Integrity of the tear film of the eye}

A study conducted by Montés-Micó et al. ${ }^{45}$ has shown that tear film irregularities appear to contribute to overall ocular HOAs. If WAs are thus dependent on corneal tear film irregularity, we can assume that greater irregularity of the tear film may lead to larger WAs. Montés-Micó et al. ${ }^{45}$ investigated the difference in HOAs between normal and dry eyes. The dry eye group showed an increase of up to 2.5 times for coma, spherical and total HOAs compared with normal eyes (in photopic and scotopic conditions). Coma and SA contributed roughly equally to the HOAs in both dry and normal eyes (photopic and scotopic conditions). The overall WAs were found roughly two times greater in scotopic conditions compared with photopic conditions, for both dry and normal eyes and the RMS Zernike coefficients for dry eyes were found to be almost double that of normal eyes. Asymmetry in the vertical meridian of the anterior surface of the corneas between two eyes would be expected as a result of gravitational effects on the tear film, and thus, greater values were found in vertical coma $\left(C_{3}^{-1}\right)$ compared with horizontal coma $\left(C_{3}^{1}\right)$, especially in the dry eyes. A greater amount of SAs were found in dry eyes compared with normal eyes, possibly due to the tendency of the tear film to thin at the centre of the cornea compared with the periphery at different rates in each subject. It is known that a thinner central tear film introduces more positive SA. Dry eyes have thus been shown by Montés-Micó et al. ${ }^{45}$ to have greater optical aberration values compared to that of normal eyes. Another study investigating patients with dry eye disease was done by Denoyer et $a .^{46}$ and the study found similar results.

In summary, tear film irregularities contribute to overall ocular HOAs, and thus, greater irregularity of the tear film may lead to larger WAs. Dry eye patients thus seem to present with higher corneal and ocular HOAs compared to eyes with a normal tear film. ${ }^{45,46}$ Studies involving ocular aberrations should thus take the ocular tear film into careful consideration to avoid misleading findings.

\section{Conclusion}

In conclusion, a variety of factors determine the accuracy of measurements taken with an aberrometer of which the most important are pupil diameter and pupil centre alignment. All of these factors must be carefully considered within a sample before data may be analysed and used for comparison among groups.

\section{Acknowledgements Competing interests}

The authors declare that they have no financial or personal relationships which may have inappropriately influenced them in writing this article.

\section{Authors' contributions}

M.O. did the literature review as part of her postgraduate studies towards the degree DPhil (Optometry). W.D.H.G. was the main supervisor during the study and also assisted in editorial corrections of the article. A.R. was co-supervisor of the study and also assisted in editorial corrections of the article.

\section{References}

1. Paquin MP, Hamam $H$, Simonet P. Objective measurement of optical aberrations in myopic eyes. Optom Vis Sci. 2002;79:285-291. http://dx.doi.org/10.1097/ 00006324-200205000-00007

2. Saw S, Katz J, Schein OD, Chew S, Chan T. Epidemiology of myopia. Epidem Rev. 1996 [cited 2014 Mar 7]; 18. Available from: http://epirev.oxfordjournals.org

3. Atchison DA, Schmid KL, Pritchard N. Neural and optical limits to visual performance in myopia. Vision Res. 2006;46:3707-3722. http://dx.doi.org/10.1016/j.visres.2006. 05.005

4. Tian Y, Tarrant J, Wildsoet CF. Optical and biometric characteristics of anisomyopia in human adults. Ophthal Physiol Opt. 2011;31:540-549. http://dx.doi.org/ 10.1111/j.1475-1313.2011.00858.x

5. Atchison DA. Recent advances in representation of monochromatic aberrations of human eyes. Clin Exp Optom. 2004;87:138-148. http://dx.doi.org/10.1111/ j.1444-0938.2004.tb03166.x

6. Buehren T, Collins MJ, Carney LG. Near work induced wavefront aberrations in myopia. Vision Res. 2005;45:1297-1312. http://dx.doi.org/10.1016/j.visres. 2004.10.026

7. Atchison DA. Optical models for human myopic eyes. Vision Res. 2006;46:22362250. http://dx.doi.org/10.1016/j.visres.2006.01.004

8. Thibos LN, Bradley A, Liu T, Lopez-Gil N. Spherical aberration and the sign of defocus. Optom Vis Sci. 2013;90:1284-1291. http://dx.doi.org/10.1097/OPX. 0000000000000040

9. Gwiazda J, Thorn F, Bauer J, Held R. Emmetropization and the progression of manifest refraction in children followed from infancy to puberty. Clin Vis Sci. 1993;8:337-344.

10. Parnissen O. Astigmatism and school myopia. Acta Ophthalmologica. 1991:69:786-790.

11. Kwan WC, Yip SP, Yap MK. Monochromatic aberrations of the human eye and myopia. Clin Exp Optom. 2009;92:304-312. http://dx.doi.org/10.1111/j. 1444-0938.2009.00378.x

12. Karimian F, Feizi S, Doozande A. Higher-order aberrations in myopic eyes. J Ophthalmic Vis Res. 2010;5:3-9.

13. Wang L, Koch DD. Ocular higher-order aberrations in individuals screened for refractive surgery. J Cat Refract Surg. 2003;29:1896-1903. http://dx.doi. org/10.1016/S0886-3350(03)00643-6

14. Porter J, Guirao A, Cox IG, Williams DR. Monochromatic aberrations of the human eye in a large population. J Opt Soc Am A Opt Image Sci Vis. 2001;18:1793-1803. http://dx.doi.org/10.1364/JOSAA.18.001793

15. Castejón-Mochón JF, López-Gil N, Benito A, Artal P. Ocular wave-front aberration statistics in a normal young population. Vision Res. 2002;42:1611-1617. http:// dx.doi.org/10.1016/S0042-6989(02)00085-8

16. Carkeet A, Leo SW, Khoo BK, Au Eong KG. Modulation transfer functions in children: Pupil size dependence and meridional anisotropy. Invest Ophthalmol Vis Sci. 2003;44:3248-3256. http://dx.doi.org/10.1167/iovs.02-1064

17. Wei RH, Lim L, Chang WK, Tan DTH. Higher order aberrations in eyes with myopia in a Chinese population. J Refract Surg. 2006;22:695-702.

18. He JC, Sun P, Held R, Thorn F, Sun X, Gwiazda JE. Wavefront aberrations in eyes of emmetropic and moderately myopic school children and young adults. Vision Res. 2002;42:1063-1070. http://dx.doi.org/10.1016/\$0042-6989(02)00035-4

19. Levy Y, Segal O, Avni I, Zadok D. Ocular higher-order aberrations in eyes with supernormal vision. Am J Ophthalmol. 2005;139:225-228. http://dx.doi org/10.1016/j.ajo.2004.08.035

20. Li T, Zhou X, Chen Z, Zhou X, Chu R, Hoffman MR. Relationship between ocular wavefront aberrations and refractive error in Chinese school children. Clin Exp Optom. 2012;95:399-403. http://dx.doi.org/10.1111/j.1444-0938.2012.00739.x

21. Carkeet A, Luo HD, Tong L, Saw SM, Tan DTH. Refractive error and monochromatic aberrations in Singaporean children. Vision Res. 2002;42:1809-1824. http://dx. doi.org/10.1016/S0042-6989(02)00114-1

22. Collins MJ, Wildsoet CF, Atchison DA. Monochromatic aberrations and myopia. Vision Res. 1995;35:1157-1163. http://dx.doi.org/10.1016/0042-6989(94)00236-F

23. Cheng $X$, Bradley A, Hong $X$, Thibos LN. Relationship between refractive error and monochromatic aberrations of the eye. Optom Vis Sci. 2003;80:43-49. http:// dx.doi.org/10.1097/00006324-200301000-00007

24. Hartwig A, Atchison DA. Analysis of higher-order aberrations in a large clinical population. Invest Ophthalmol Vis Sci. 2012;53:7862-7870. http://dx.doi. org/10.1167/iovs.12-10610 
25. Gwiazda J, Grice K, Held R, McLellan J, Thorn F. Astigmatism and the development of myopia in children. Vis Res. 2000:40:1019-1026. http://dx.doi.org/10.1016/ S0042-6989(99)00237-0

26. Campbell FW, Green DG. Optical and retinal factors affecting visual resolution. J Physiol. 1965;181:576-593. http://dx.doi.org/10.1113/jphysiol.1965.sp007784

27. Williams DR, Porter J, Yoon G, et al. How far can we extend the limits of human vision? In: Krueger RR, Applegate RA, MacRae SM, editors. Wavefront customized visual correction: The quest for Super Vision II. Thorofare, NJ: Slack Inc., 2004; p. 19-38.

28. Feizi S, Karimian F. Effect of higher order aberrations on contrast sensitivity function in myopic eyes. Jpn J Ophthalmol. 2009;53:414-419.

29. Wang Y, Zhao K, Yang X, He J, Wang W. Higher order aberrations and low contras vision function in myopic eyes $(-3.00$ to $-6.00 \mathrm{D})$ under mesopic conditions. Refract Surg. 2011;27:127-134. http://dx.doi.org/10.3928/1081597X-20100430-01

30. Roorda A. A review of basic wavefront optics. In: Krueger RR, Applegate RA MacRae SM, editors. Wavefront customized visual correction: The quest for Super Vision II. Thorofare, NJ: Slack Inc., 004; p. 9-18.

31. Applegate RA, Hilmantel G, Thibos LN. Assessment of visual performance. In Krueger RR, Applegate RA, MacRae SM, editors. Wavefront customized visua correction: The quest for Super Vision II. Thorofare, NJ: Slack Inc., 2004; p. 65-76.

32. Hunter JJ, Campbell MCW, Kisilak ML, Irving EL. Blur on the retina due to higherorder aberrations: Comparison of eye growth models to experimental data. J Vis. 2009;9:1-20. http://dx.doi.org/10.1167/9.6.12

33. Rodríguez P, Navarro R, González L, Hernández JL. Accuracy and reproducibility of Zywave, Tracey, and experimental aberrometers. J Refract Surg. 2004;20:810-817.

34. Burakgazi AZ, Tinio B, Bababyan A, Niksarli MD, Asbell P. Higher order aberrations in normal eyes measured with three different aberrometers. J Refract Surg. 2006;22:898-903.

35. Ginis HS, Plainis S, Pallikaris A. Variability of wavefront aberration measurements in small pupil sizes using a clinical Shack-Hartmann aberrometer. BMC Ophthalmol. 2004;4:1. http://dx.doi.org/10.1186/1471-2415-4-1
36. Dobos MJ, Twa MD, Bullimore MA. An evaluation of the Bausch \& Lomb Zywave aberrometer. Clin Exp Optom. 2009;92:238-245. http://dx.doi.org/10.1111/ j.1444-0938.2009.00360.x

37. Smolek MK. Method for expressing clinical and statistical significance of ocular and corneal wave front error aberrations. Cornea. 2012;31:212-221. http:// dx.doi.org/10.1097/ICO.0b013e318221ce7d

38. López-Gil N, Fernández-Sánchez V, Legras R, Montés-Micó R, Lara F, Nguyen-Khoa $\mathrm{JL}$. Accommodation-related changes in monochromatic aberrations of the human eye as a function of age. Invest Ophthalmol Vis Sci. 2008;49:1736-1743. http:// dx.doi.org/10.1167/iovs.06-0802

39. Atchison DA, Collins MJ, Wildsoet CF, Christensen J, Waterworth MD. Measurement of monochromatic ocular aberrations of human eyes as a function of accommodation by the Howland aberroscope technique. Vision Res. 1995;35:313-323. http://dx.doi.org/10.1016/0042-6989(94)00139-D

40. Awwad ST, Warmerdam D, Lee D, Bowman RW, Cavanagh HD, McCulley JP. Letters to the Editor: Changes in ocular higher order aberrations with accommodation in wavefront-guided LASIK myopic candidates. J Refract Surg. 2009;25:171-172.

41. Atchison DA, Markwell EL. Aberrations of emmetropic subjects at different ages. Vis Res. 2008;48:2224-2231. http://dx.doi.org/10.1016/j.visres.2008.06.023

42. Jahnke M, Wirbelauer C, Pham DT. Influence of age on optical aberrations of the human eye. Ophthamologe. 2006;103:596-604. http://dx.doi.org/10.1007/ s00347-006-1361-4

43. Taneri S, Oehler S, Azar DT. Influence of mydriatic eye drops on wavefront sensing with the Zywave Aberrometer. J Refract Surg. 2011;27:678-685. http://dx.doi.org /10.3928/1081597X-20110317-01

44. Tabernero J, Atchison DA, Markwell EL. Aberrations and pupil location under corneal topography and Hartmann-Shack illumination conditions. Invest Ophthalmol Vis Sci. 2009;50:1964-1970. http://dx.doi.org/10.1167/iovs.08-2111

45. Montés-Micó R, Cáliz A, Alió JL. Wavefront analysis of higher order aberrations in dry eye patients. J Refract Surg. 2004;20:243-247.

46. Denoyer A, Rabut G, Baudouin C. Tear film aberration dynamics and vision-related quality of life in patients with dry eye disease. Ophthalmol. 2012;119:1811-1818. $\mathrm{http}: / / \mathrm{dx}$.doi.org/10.1016/j.ophtha.2012.03.004 